\title{
A Fast Handoff Mechanism for IEEE 802.11 and IAPP Networks
}

\author{
Ping-Jung Huang \\ Computer \& Communications Research Laboratories \\ Industrial Technology Research Institute \\ Hsin-Chu, 310, Taiwan \\ E-Mail: pjhuang.eic92g@nctu.edu.tw
}

\author{
Yu-Chee Tseng \\ Department of Computer Science \\ National Chiao-Tung University \\ Hsin-Chu, 300, Taiwan \\ E-Mail: yctseng@csie.nctu.edu.tw
}

\begin{abstract}
Handoff is a critical issue in IEEE 802.11-based wireless networks. In this paper we propose a fast and seamless handoff solution for IEEE 802.11 wireless LAN with IAPP. It is based on a concept of neighbor graph, which describes the nearby access points (APs) that a mobile host (MH) may find. Then we further derive selective scanning with unicast in power-save mode, pre-registration of IAPP, and frame forwarding-and-buffering mechanisms. Selective scanning allows a MH to only try potential handoff targets. Pre-registration allows early transfer of a MH's security context from its old AP to new AP. The forwarding-and-buffering mechanism is to solve the packet loss problem during handoff. Our performance evaluation shows that the proposed solution can result in $\mathbf{9 0 \%}$ reduction in the handoff latency from standard handoff procedure.
\end{abstract}

\section{INTRODUCTION}

Recently, the IEEE 802.11 wireless LAN (WLAN) [1] has grown rapidly due to its easy operation, low cost, and high throughput. Many applications, such as Voice over IP (VoIP), instant message, and media-streaming services, have been proposed to run on top of WLANs.

However, handoff, an inherent problem with wireless networks, especially for real-time applications, has not been well addressed in IEEE 802.11. IEEE 802.11 takes a hard handoff approach, which means that a mobile host $(\mathrm{MH})$ has to break its connection with its old access point (AP) before connecting to a new AP. This may result in long handoff latency. According to [2], it is found that the handoff procedure in IEEE 802.11 normally takes hundreds of milliseconds and almost $90 \%$ of the handoff delay is due to search of new APs, or so-called the probe delay. This is unsatisfactory because, for example, the recommended maximum handoff latency for VoIP applications is $50 \mathrm{~ms}$ [3].

In this paper, we propose a fast and seamless mechanism for IEEE 802.11 networks that support IAPP. During handoff, to select the next AP, a MH does not scan all channels. Instead, it only selectively scans some potential APs with unicast based on the neighbor graph (NG) provided by a NG Server [4]. We enhance the NG approach [4] by putting the $\mathrm{MH}$ to power-saving mode to pre-scan neighboring APs. Then we further derive selective scanning with unicast in power-save mode, pre-registration of IAPP, and frame forwarding-and-buffering mechanisms. Selective scanning allows a $\mathrm{MH}$ to only try potential handoff targets. Pre-registration allows early transfer of a MH's security context from its old AP to new AP. The forwarding-and-buffering mechanism is to solve the packet loss problem during handoff.

Section 2 reviews related work. We introduce our fast and seamless handoff mechanism in Section 3. Performance issues are discussed in Section 4. Conclusions are drawn in Section 5 .

\section{RELATED WORK}

\section{A. IEEE 802.11 Handoff Schemes}

IEEE 802.11 standard [1] defines two operation modes: infrastructure and ad hoc modes. In the infrastructure mode, an AP comprises a Basic Service Set (BSS) and provides connectivity to networks for their associated MHs. One or more APs comprise an Extended Service Set (ESS) to cover a larger area. In ad hoc mode, two or more MHs can form a peer-to-peer network without AP.

An ideal WLAN provides successive radio signal coverage for MHs in its service area. A MH may decide to handoff from an AP to another AP due to mobility, loading of AP, or fading of signals. The handoff procedure of IEEE 802.11 can be divided into two steps: discovery and reauthentication [2].

1) Discovery: In order to find a nearby AP, a MH scans all channels either passively or actively. In passive scanning, a MH listens to APs' periodic beacon messages to know their parameters, such as beacon interval, capability information, BSSID, supported rate, etc. The period of beacon frames is normally set to $100 \mathrm{~ms}$ in most implementations. In active scanning, for every channel, a $\mathrm{MH}$ will broadcast a probe request and expect probe responses from APs.

In active scanning, the scanning delay can be calculated as [4]: 


$$
N_{c h} \cdot T_{b} \leq t \leq N_{c h} \cdot T_{t}
$$

Where $\mathrm{N}_{\mathrm{ch}}$ is the total number of channels (normally, $\mathrm{N}_{\mathrm{ch}}=12$ for $802.11 \mathrm{a}$ and $\mathrm{N}_{\mathrm{ch}}=11$ for $\left.802.11 \mathrm{~b} / \mathrm{g}\right), \mathrm{T}_{\mathrm{b}}=$ MinChannelTime is the minimum time that a $\mathrm{MH}$ has to wait on a channel if no response is received, and $\mathrm{T}_{\mathrm{t}}=$ MaxChannelTime is the maximum time that a $\mathrm{MH}$ has to wait on a channel if responses are received. In most implementations, the MaxChannelTime is set to $30 \mathrm{~ms}$, which implies that the worst delay of active scanning, for example, in IEEE $802.11 \mathrm{~b}$ is $300-400 \mathrm{~ms}$. An experimental result can be found in [4].

2) Reauthentication: This typically involves the authentication and reassociation procedures. The reauthentication phase transfers the credentials of the $\mathrm{MH}$ from the old AP to the new AP. IEEE 802.11 defines two subtypes of authentication service: 1) Open System, which is a null authentication algorithm and 2) Shared Key, which is a four-way authentication mechanism. If IAPP is used, only null authentication frames need to be exchanged in the reauthentication phase. In our experience, exchanging null authentication frames takes about 1-2 ms.

After authentication, the reassociation process involves exchanging reassociation request, and reassociation response frames. From our experience, the reassociation delay takes around 1-2 ms. In IEEE 802.11 with IAPP networks, additional IAPP messages between the old AP and the new AP will increase the reassociation latency to $40 \mathrm{~ms}$ [5].

Fig. 1 summarizes the handoff procedure. The overall latency is more than $300 \mathrm{~ms}$ (including IAPP message overhead). The probe delay constitutes the biggest part (over $90 \%$ ) of the delay [2]. The objective of our proposal is to reduce the handoff latency to less than $50 \mathrm{~ms}$ in order to support most of real-time applications.

To reduce the handoff latency, many approaches $[4,10,11$, $12,13]$ have been proposed. Reference [4], a concept called neighbor graph $(N G)$ is proposed. From the NG provided by an external server, a $\mathrm{MH}$ only needs to scan the channels that are used by its current AP's neighbors. According to [4], about $10 \mathrm{~ms}$ are needed to scan a specific neighbor. In this paper, we will propose a selective scanning mechanism to farther reduce the search time. Reference [10] shows how to calculate the optimal MaxChannelTime, MinChannelTime, and beacon interval. However, it still has to scan all channels.

Reference [11], it is suggested that when scanning a channel, a MH will evaluate the number of APs (say Y) in that channel by monitoring packets sent in that channel. The $\mathrm{MH}$ will stop scanning that channel whenever it has collected probe responses from at least $\mathrm{Y}$ APs so as to reduce the scanning time. However, the scheme still has to scan all channels.

Some fast handoff solutions focus on bypassing the scanning phase [12] [13]. Reference [12], a MH maintains a cache which contains a list of APs adjacent to its current AP. The cached data was established from its previous scanning. Only the two APs with the best received signal strength indication (RSSI) were cached. During handoff, the cached

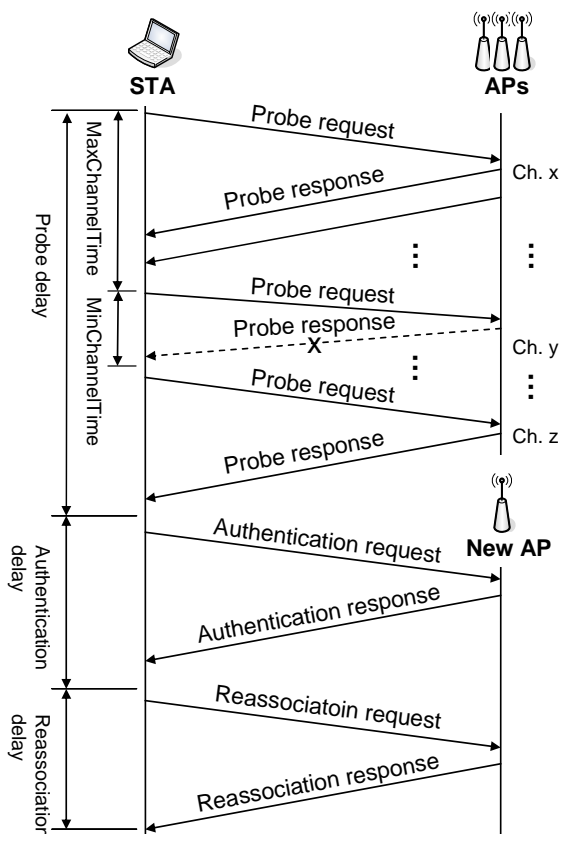

Figure 1. IEEE 802.11 handoff procedure.

APs are searched first. If this fails, a selective scanning is performed. However, when the cache is obsolete, scanning is still inevitable. Reference [13], a MH can predict its movement path and select the potential AP. A location server can provide information about APs so that a $\mathrm{MH}$ can re-associate with its new AP directly without going through the probe procedure. However, this scheme relies on a precise localization method.

\section{B. Inter Access Point Protocol (IAPP)}

To reduce the opportunity of transmitting security information of MHs in the air during the handoff period, IAPP proposes to allow an AP to communicate with other APs to exchange relevant information of associated MHs on a common DS. An IEEE 802.11 with IAPP network typically comprises APs, MHs, and Remote Authentication Dial In User Service (RADIUS) servers [14]. The RADIUS servers provide two functions: 1) mapping of the BSSID of an AP to its IP address on the distribution system medium (DSM) and 2) distribution of keys to APs to allow secure communications between APs [6].

The handoff procedure in IAPP is illustrated in Fig. 2. When a MH moves away from its current AP, it may start to search for a new AP by active or passive scanning. If a new AP is located, the $\mathrm{MH}$ will send a reassociation request frame to the new AP. The request frame contains MH's MAC address and the BSSID of the old AP. Upon receiving the reassociation request frame, the new AP sends a RADIUS Access-Request packet to the RADIUS server to verify the old BSSID. If the old AP is valid, the RADIUS server will reply to the new AP a RADIUS Access-Accept packet which contains a security block for communication between the old AP and the new AP. Then the new AP will send an encrypted IAPP MOVE-notify packet to the old AP, which will reply an encrypted IAPP 


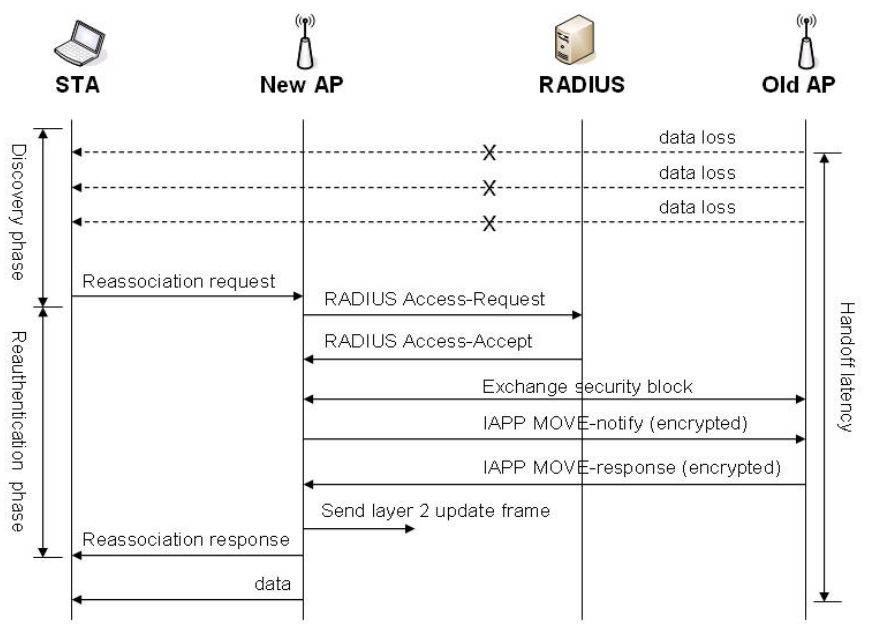

Figure 2. The handoff procedure in IAPP.

MOVE-response packet with the context information pertaining to the $\mathrm{MH}$. Upon receiving the IAPP MOVE-response packet, the new AP will broadcast a layer-2 update frame to the DS to inform all layer-2 devices to update their forwarding information about this $\mathrm{MH}$. Finally, the new AP will send the $\mathrm{MH}$ a reassociation response frame. This completes the handoff procedure. To conclude, IAPP can avoid transferring the MH's security information in the air but does not reduce the handoff latency effectively. According to [5], transferring a MH's context takes about $40 \mathrm{~ms}$.

Considering that IAPP may cause frame loss during handoff, [15] proposes to add a layer-2 frame buffering-and-forwarding mechanism to IAPP. Each buffered layer-2 frame at the old AP can be carried by a new IAPP MOVE-forward packet to the new AP, following the IAPP MOVE-response packet. Thus, this enables seamless handoff between APs.

\section{The PROPOSED FAST AND SEAMLESS HANDOFF MECHANISM}

We propose a fast handoff mechanism by combining an enhanced neighbor graph scheme and an enhanced IAPP scheme. As mentioned earlier, the greater part of handoff latency is probe delay. If a MH knows exactly its adjacent APs, it can use selective scanning by unicast to avoid scanning all channels. Fig. 3 shows the system architecture. The dotted lines represent neighborhood relationship of APs. There is a NG Server in the IEEE 802.11 network. Every MH runs an application-level called NG Client responsible for exchanging NG information with the NG Server. The NG Server maintains a NG table, as shown in Table I. Each entry of the NG table indexed by the MH's current AP contains 32 bytes of a neighbor SSID, 1byte of channel, 1 byte of loading, 4 bytes of IP address, and 6 bytes of BSSID. The NG table can be set manually or can be collected from roaming history of MHs (such as reassociation requests). The loading filed is an optional field to represent the number of MHs currently associated with an AP. It can be used to select a light-load AP. (The issue is irrelevant to the theme of this paper and is thus ignored below.)

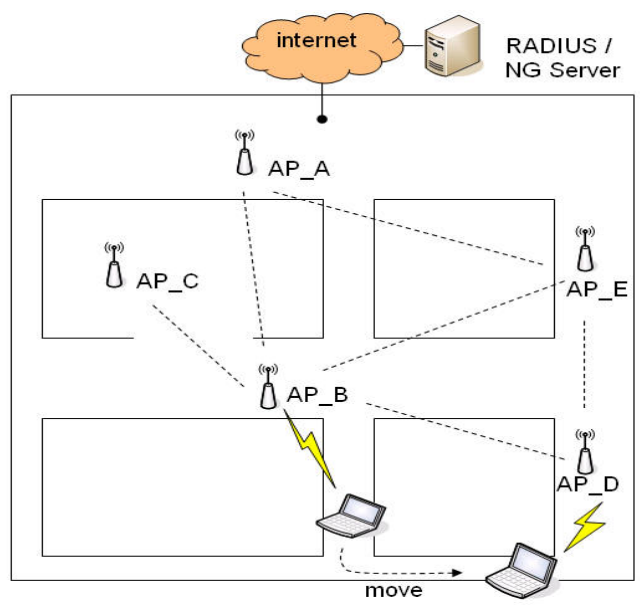

Figure 3. The system architecture of our fast and seamless handoff mechanism.

TABLE I. NG TABLE

\begin{tabular}{|c|c|c|c|c|c|}
\hline Current AP & Neighbor & Ch. & Loading & IP & BSSID \\
\hline \multirow{3}{*}{ AP_A } & AP_B & 6 & 2 & $192.168 \ldots$ & $00: 60: \mathrm{B} 3 \ldots$ \\
\cline { 2 - 6 } & AP_E & 6 & 6 & $192.168 \ldots$ & $00: 60: \mathrm{B} 3 \ldots$ \\
\cline { 2 - 6 } & AP_D & 11 & 7 & $192.168 \ldots$ & $00: 60: \mathrm{B} 3 \ldots$ \\
\cline { 2 - 6 } & $\ldots$ & $\ldots$ & $\ldots$ & $\ldots$ & $\ldots$ \\
\hline \multirow{3}{*}{ AP_B } & AP_A & 1 & 3 & $192.168 \ldots$ & $00: 60: \mathrm{B} 3 \ldots$ \\
\cline { 2 - 6 } & AP_C & 11 & 1 & $192.168 \ldots$ & $00: 60: \mathrm{B} 3 \ldots$ \\
\cline { 2 - 6 } & AP_D & 11 & 7 & $192.168 \ldots$ & $00: 60: \mathrm{B} 3 \ldots$ \\
\cline { 2 - 6 } & $\ldots$ & $\ldots$ & $\ldots$ & $192.168 \ldots$ & $\ldots$ \\
\hline
\end{tabular}

The original IAPP provides several functions to support host mobility, known as post-registration. However, there exists a period of handoff time, during which the MH cannot send/receive any frame to/from APs. These frames may be lost. Therefore, we propose a pre-registration mechanism for IAPP to reduce the handoff latency. To avoid losing frames, we propose a frame forwarding-and-buffering scheme. Six new IAPP packets are designed for this purpose.

Fig. 4 shows the overall message flow in our handoff scheme. The state machine of the NG Client is illustrated in Fig. 5.

1) When a $\mathrm{MH}$ associates with our network, its NG Client automatically connects to the NG Server to get the portion of the NG table indexed by its current AP. This gives the MH the neighbors near its current AP. These neighbors will be the target of the selective scanning procedure in step 2. Initially, the NG Client is in the idle state.

2) Periodically, the MH will check the RSSI of its current AP. when the RSSI of its current AP is less than Threshold scan, $_{\text {, }}$ the NG Client will enter the selective scanning and pre-registration state. In this state, the $\mathrm{MH}$ will notify its 


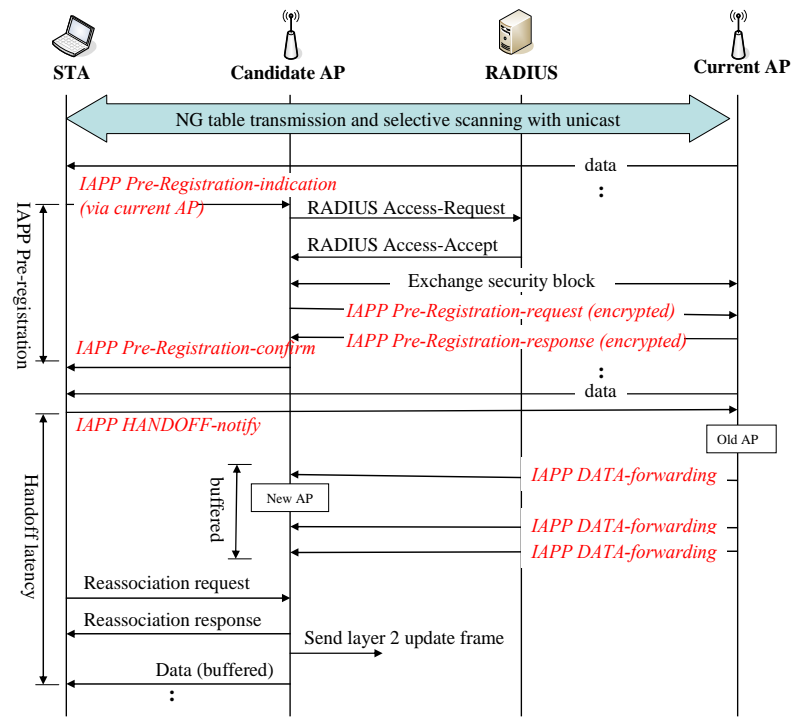

Figure 4. The proposed pre-registration and forwarding-and-buffering mechanisms for IAPP.

current AP that it will enter the power-saving mode so that the AP can buffer incoming data for the MH. During this period, the MH will scan each all neighboring AP collected from step 1 by unicasting a probe request. The time that the $\mathrm{MH}$ should wait for a response from each AP is no more than MinChannelTime. If there is neither response nor traffic in that channel during MinChannelTime, the probe is regarded failure and the next AP is probed. This is known as selective scanning. After finishing scanning all APs, the $\mathrm{MH}$ will inform its current AP that it has returned to the active mode to receive data.

3) After returning to the active mode, the NG Client will send an IAPP Pre-Registration-indication packet to the candidate AP that has the best RSSI in step 2's probing via its current AP. Upon receiving the IAPP Pre-Registration-indication packet, the candidate AP will send a RADIUS Access-Request packet to the RADIUS server to verify the current AP's BSSID obtained from the received IAPP Pre-Registration-indication packet. If the current AP is legal, the RADIUS server will reply a RADIUS Access-Accept packet containing a security block for communication between the current AP and the candidate AP. On receiving the RADIUS Access-Accept packet, the candidate AP will exchange a security block with the current AP. After that, the candidate AP will send an encrypted IAPP Pre-Registration-request packet to the current AP to request the context information of the MH. In return, the current AP will reply an IAPP Pre-Registration-response packet which includes the MH's context information. Then the candidate AP will respond an IAPP Pre-Registration-confirm packet to the $\mathrm{MH}$. This completes the pre-registration procedure. Note that this would allow the candidate AP to accept an association request from the $\mathrm{MH}$ directly without further going through the probe procedure.

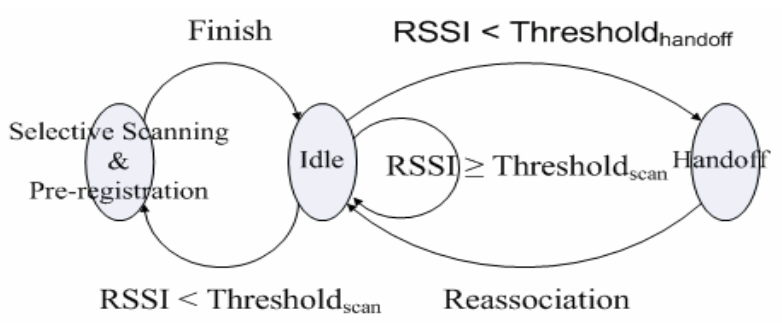

PS. Threshold scan $>$ Threshold $_{\text {handoff }}$

Figure 5. The state machine of NG Client.

4) When the RSSI of the current AP sensed is continuously decreasing and becomes less than Threshold ${ }_{\text {handoff, the NG }}$ Client will make a handoff decision and enter the handoff state. The NG Client will then notify the current AP the fact by sending it an IAPP HANDOFF-notify packet containing the ID of the new AP. The current AP will break its association with the $\mathrm{MH}$. Then the $\mathrm{MH}$ can directly reassociate with the new AP. Before the old AP receives the corresponding layer-2 update frame, all frames for the $\mathrm{MH}$ will still be routed to the old AP. These frames will be forwarded to the new AP by IAPP DATA-forwarding packets. Upon receiving these frames, the new AP will buffer these frames and deliver them to the $\mathrm{MH}$ after the $\mathrm{MH}$ (re)associates with the new AP. The NG Client will then return to the idle state.

5) After the MH handoffs to the new AP, the NG Client will automatically connect to the NG Server to get the portion of the NG table related to its new AP. So it is prepared for the next handoff.

Fig. 6 (a) summarizes the handoff life cycle under the proposed scheme. Fig. 6 (b) shows the change of signal strengths between two APs during handoff.

\section{PERFORMANCE DisCUSSION}

In the section, we evaluate the performance of our approach. For the selective scanning, its latency can be written as

$$
T_{\text {scan }}=\text { MinChannelTime } \times N \text {. }
$$

According to [10], the optimum MinChannelTime is 1 TU (1024 $\mu \mathrm{s}$ ), and here we believe that $3 \mathrm{~ms}$ should be sufficient. If a hexagonal cellular AP deployment is adopted, then $\mathrm{N}$ is about 6 , which means that $\mathrm{T}_{\text {scan }}=18 \mathrm{~ms}$. This should satisfy the timing constraint of most VoIP applications. In contrast, the typical handoff latency in an IEEE 802.11b with IAPP network may take a probe delay of $\mathrm{t}_{\text {probe }}=40 \sim 300 \mathrm{~ms}$ and an IAPP delay of $\mathrm{t}_{\text {IAPP }}=40 \mathrm{~ms}$ [5]. This would exceed the recommended latency of $50 \mathrm{~ms}$ in VoIP applications. In our approach, the pre-registration mechanism would eliminate the $t_{\text {IAPP }}$ part. The $t_{\text {probe }}$ part is reduced to $T_{\text {scan }}$. The handoff latency only contains the reassociation delay, which should be bounded within 2 ms. 


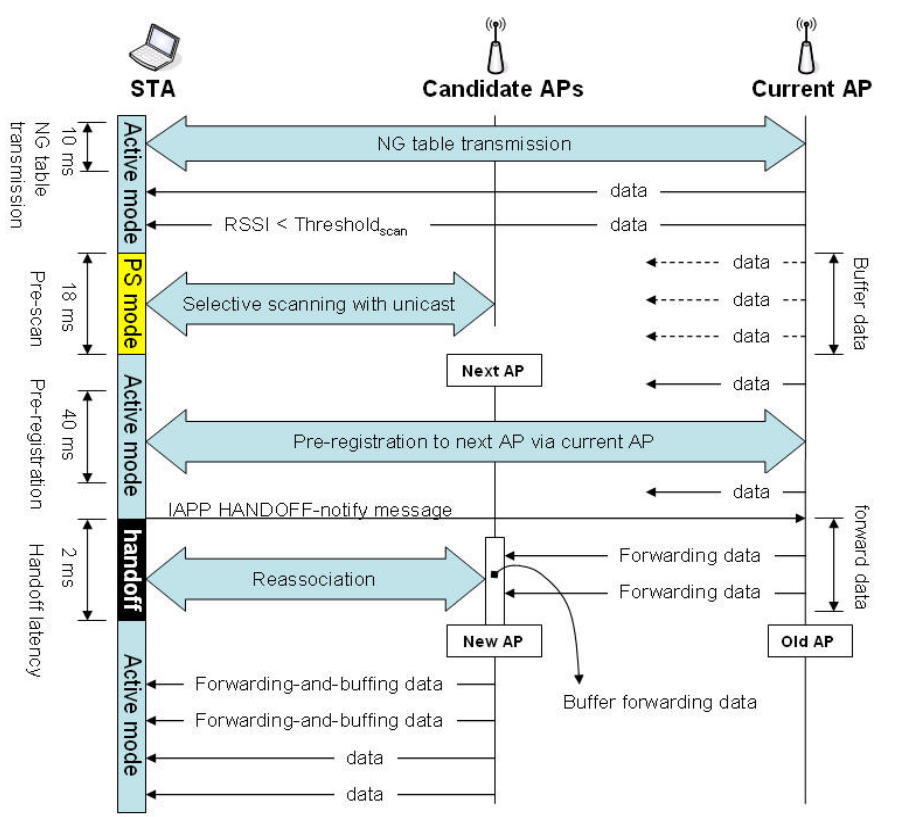

(a)

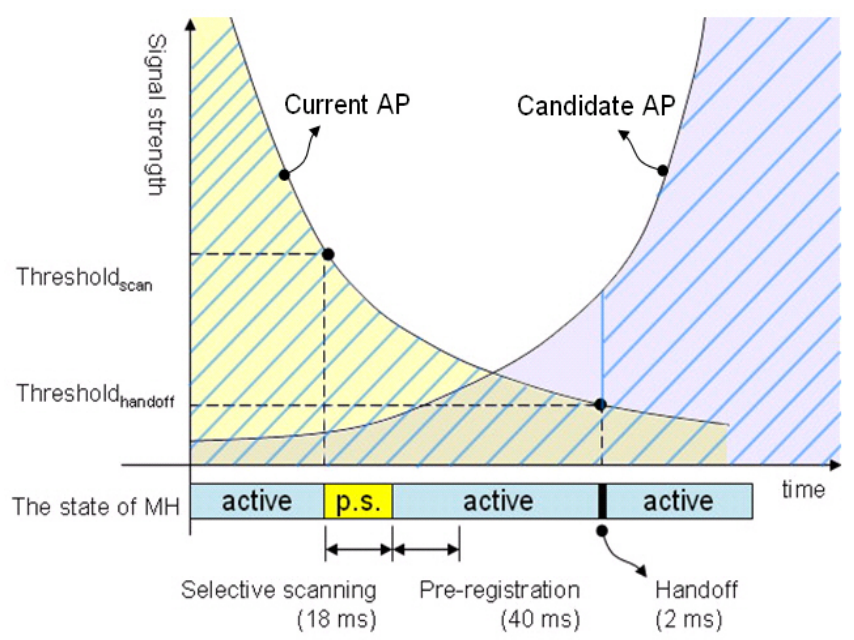

(b)

Figure 6. (a) Handoff life cycle and (b) change of signal strengths during handoff.

\section{CONCLUSION}

In this paper, we have proposed a fast and seamless handoff mechanism for IEEE 802.11 network with IAPP. Many approaches have been proposed for the selection of the next AP or improvement of IAPP. In our approach, we tightly integrate the handoff procedure with the IAPP context-switching procedure. Most of the operations related to handoff are executed before handoff, including the selection of the next AP and the transfer of MH's context. By switching a $\mathrm{MH}$ to the power-saving mode to process these operations, our mechanism actually achieves a certain degree of soft handoff. We have also proposed a frame forwarding-and-buffering mechanism to avoid losing data during handoff. The result is a reduction of $90 \%$ of handoff latency from typical procedure. It should be able to support most VoIP applications without problem.

\section{REFERENCES}

[1] "Wireless LAN Medium Access Control (MAC) and Physical Layer (PHY) Specifications,” IEEE Standard, 1999.

[2] Mishra, A Shin, and W. Arbaugh, "An Empirical Analysis of the IEEE 802.11 MAC Layer Handoff Process,” ACM SIGCOMM Computer Comm. Rev., vol. 33, no. 2, Apr. 2003.

[3] International Telecommunication Union, "General Characteristics of International Telephone Connections and International Telephone Circuits," ITU-TG.114, 1988.

[4] H. Kim, S. Park, C. Park, J. Kim, and S. Ko, "Selective Channel Scanning for Fast Handoff in Wireless LAN using Neighbor Graph”, ITC-CSCC 2004, July 2004.

[5] B. Aboba, "Fast handoff issues," IEEE-03-155r0-I, IEEE 802.11 Working Group, Mar. 2003.
[6] "IEEE Trial-Use Recommended Practice for Multi-Vendor Access Point Interoperability via an Inter-Access Point Protocol Across Distribution Systems Supporting IEEE 802.11 Operation", IEEE Standard, 2003.

[7] "Wireless LAN Medium Access Control (MAC) and Physical Layer (PHY) specifications High-speed Physical Layer in the 5 GHz Band" IEEE Standard, 1999.

[8] "Wireless LAN Medium Access Control (MAC) and Physical Layer (PHY) specifications: Higher-Speed Physical Layer Extension in the 2.4 GHz Band" IEEE Standard, 1999.

[9] "Wireless LAN Medium Access Control (MAC) and Physical Layer (PHY) specifications Amendment 4: Further Higher Data Rate Extension in the $2.4 \mathrm{GHz}$ Band” IEEE Standard, 2003.

[10] Velayos, H. and G. Karlsson, "Techniques to Reduce IEEE 802.11b MAC Layer Handover Time", Laboratory for Communication Networks, KTH, Royal Institute of Technology, Stockholm, Sweden, TRITA-IMIT-LCN R 03:02, April 2003.

[11] Kyoungnam Kwon and Chaewoo Lee , "A Fast Handoff Algorithm using Intelligent Channel Scan for IEEE 802.11 WLANs”, IEEE 6th International Conference, vol. 1, pp. 46- 50, 2004.

[12] S. Shin, A. S. Rawat, H. Schulzrinne, "Reducing MAC Layer HandoffLatency in IEEE 802.11 Wireless LANs", ACM MobiWac'04, Oct, 2004.

[13] C.C. Tseng, K.H. Chi, M.D. Hsieh, and H.H. Chang, "Location-based fast handoff for 802.11 networks”, IEEE Communications letters, vol. 9, issue 4, pp. 304- 306, April 2005.

[14] C. Rigney et al., "Remote Authentication Dial In User Service (RADIUS),” RFC 2058, Jan. 1997.

[15] C.T. Chou and K.G. Shin, "An Enhanced Inter-Access Point Protocol for Uniform Intra and Intersubnet Handoffs”, IEEE Transactions on Mobile Computer, vol. 4, no. 4, July/August 2005.

[16] J. Malinen, "Host AP Driver for Intersit Prism2/2.5/3," http://hostap.epitest.fi/, 2005 